\title{
Perfiles de desempeño académico: la importancia de las expectativas familiares
}

\author{
Mónica Bravo Sanzana, ${ }^{*}$ Sonia Salvo, ${ }^{* *}$ Manuel Mieres, ${ }^{* *}$ \\ Juan Mansilla, ${ }^{* * * *}$ Christian Hederich*****
}

Perfiles Latinoamericanos, 25(50)

2017 | pp. 361-386

DOI: $10.18504 / \mathrm{pl} 2550-016-2017$

\begin{abstract}
Resumen
El presente artículo explora algunos de los factores que explican el desempeño escolar. Para ello se identificaron y caracterizaron perfiles de estudiantes chilenos de octavo grado de primaria (media 13.65 años de edad/DS 0.74), en función de su desempeño académico en la prueba de Historia, Geografía y Ciencias Sociales, y variables de contexto. La base de datos fue generada por el Sistema de Medición de la Calidad de la Educación de Chile (Simce). La investigación se orientó desde el enfoque cuantitativo correlacional predictivo, utilizando el árbol de clasificación y regresión (CART). Las altas expectativas educacionales de la familia son el factor diferenciador más importante en el rendimiento escolar. Se discuten los resultados en función de la investigación y literatura generada acerca de estos temas.
\end{abstract}

\begin{abstract}
This study explored some of the factors that explain school performance. To attain this, Chilean students' profiles of eighth year of primary school were identified and characterized (13.65 year old mean/DS 0.74). This was done according to their academic performance in the History, Geography and Social Sciences test, and the context variables. The database was provided by the Sistema de Medición de la Calidad de la Educación de Chile (Simce). The study was conducted by means of a predictive correlational quantitative design, using a classification and regression tree (CART). Parents' high educational expectations are the most important distinguishing factor in school performance. The results are discussed in relation to previous research about these topics.

Palabras clave: desempeño escolar, escuela primaria, rol de la familia, expectativas educacionales de la familia, análisis de regresión, perfiles de estudiantes, CART.

Keywords: School performance, primary school, role of the family, educational expectations of parents, regression analysis, student profiles, CART.

* Doctora en Educación, Universidad de La Frontera. Núcleo Científico-Tecnológico en Ciencias Sociales Aplicadas, Universidad de La Frontera | monicaviviana38@gmail.com

** Doctora en Estadística Multivariante, Universidad de Salamanca. Departamento de Matemática y Estadística, Universidad de La Frontera | sonia.salvo@ufrontera.cl

*** Magíster en Ciencias Sociales Aplicadas, Universidad de La Frontera. Facultad de Educación, Universidad Católica de Temuco | mieres@uctemuco.cl

**** Doctor en Filosofía y Letras, Universidad Pontificia de Salamanca. Facultad de Educación, Universidad Católica de Temuco | jmansilla@uct.cl

***** Doctor en Psicológica, Universidad Autónoma de Barcelona. Departamento de Psicopedagogía, Universidad Pedagógica Nacional | christian.hederich@gmail.com
\end{abstract}




\section{Introducción ${ }^{1}$}

gran debate en Chile respecto del sistema educacional se encuentra focalizado en torno a su calidad y equidad. Para ello, la información más utilizada son los resultados de aprendizaje de los estudiantes en las pruebas estandarizadas (Bellei et al., 2013; Mizala \& Torche, 2012; OECD, 2012).

En este contexto, la política educacional chilena le ha asignado a este tipo de evaluación un rol en el mejoramiento de la educación. Y lo ha hecho asociando resultados a consecuencias e incentivos, a la definición en la administración de recursos, y a clarificar las expectativas de logro a través de estándares de desempeño (Blanco, 2009; Meckes, 2007). Así, el sistema escolar chileno sitúa a la escuela como un espacio privilegiado para proveer mayores y mejores oportunidades de aprendizaje.

Los estudios evidencian que el grado de polarización académica y socioeconómica del sistema escolar chileno durante la última década ha sido constante (Villalobos \& Valenzuela, 2012). La brecha socioeconómica entre los establecimientos educacionales es alta, a pesar del mayor gasto público en educación desde la década de los noventa. Para comprender mejor esta situación educativa, es necesario referenciar las características del sistema educacional de Chile.

El origen del actual sistema escolar chileno se encuentra en la reforma educativa de los años ochenta. Fue una reforma pionera en el mundo cuyos fundamentos teóricos se encuentran en la economía de mercado y en el subsidio educacional (o voucher) por alumno (Friedman, 1955). El objetivo era descentralizar la educación y, como primera acción implementada, todas las unidades educativas administradas por el Estado quedaron bajo la administración de los municipios (Cox \& Lemaitre, 1999).

De este modo, se definieron los siguientes tipos de instituciones educativas: la escuela pública administrada por las municipalidades, la escuela particular subvencionada por el Estado y administrada por privados, la escuela particular financiada y administrada por privados, y corporaciones sin fines de lucro (MINEDUC, 2011; Paredes \& Pinto, 2009). Su participación en la matrícula hacia fines de la década de 2010 era de 37.5, 53.9, 7.1 y $1.5 \%$, respectivamente.

Cabe destacar el intenso debate respecto del crecimiento de la escuela particular subvencionada en desmedro de la educación pública. Un estudio reveló que este cambio en la composición de la matrícula en Chile se explica por factores

1 Esta investigación utilizó como fuente de información las bases de datos Simce de la Agencia de Calidad de la Educación (Ministerio de Educación). Todos los resultados del estudio son de responsabilidad de los autores y en nada comprometen a dicha institución. 
de demanda, asociados a las decisiones de los padres, y factores de oferta, asociados a las decisiones de los sostenedores, además de las restricciones asimétricas sobre financiamiento y costos en el sistema educativo (Paredes \& Pinto, 2009). En cuanto a la distribución de los estudiantes en las escuelas de Chile, una investigación señala que la misma da cuenta de la existencia de procesos de estratificación, segregación y debilidad cohesionadora en el sistema educativo chileno. La estratificación se ve reflejada en que el tipo de establecimiento educacional determina las características de los estudiantes que la integran (Villalobos \& Valenzuela, 2012).

Así, la reforma educacional chilena descentralizó la administración de las unidades educativas del sector público, promoviendo, entre otros objetivos, la competencia entre ellas. Sin embargo, el Ministerio de Educación llevó a cabo un papel centralizador en el diseño del currículo, horario escolar, y sistema de evaluación y promoción, entre otras funciones. En cuanto a la evaluación, en 1988 se creó el Sistema de Medición de la Calidad de la Educación de Chile (en adelante, Simce) (Cox \& Lemaitre, 1999). Este organismo se encarga de realizar las mediciones anuales a toda la población de estudiantes del nivel en diversos dominios y publicar sus resultados de aprendizaje.

De acuerdo a la lógica de la reforma, los padres poseen la facultad de evaluar los resultados y elegir el establecimiento educacional para sus hijos. Teóricamente, esto posibilita la competencia entre las instituciones educativas, porque subyace la creencia de que los padres son consumidores críticos de la educación (Taut et al., 2009). No obstante, hay evidencia de que esto no ocurre (Chumacero et al., 2011).

Cabe agregar que se necesita profundizar en investigaciones y experiencias que evidencien la mejora en el desempeńo escolar, en especial de aquellos estudiantes provenientes de familias socioeconómicamente vulnerables. La investigación educativa ha presentado experiencias exitosas realizadas en establecimientos educacionales vulnerables donde a través de estrategias educativas asertivas se logró mejorar el desempeño de los estudiantes (Bellei et al., 2004; García \& Paredes, 2010).

Esta investigación puede considerarse una contribución, pues indaga en factores que podrían aportar a una mejor comprensión del desempeño de los estudiantes en las pruebas Simce.

\section{Factores que explican el desempeño académico}

La reciente investigación internacional ha mostrado que existen factores claramente identificables en relación con el desempeño académico. Uno de ellos es 
la dependencia administrativa o tipo de establecimiento educacional. En este sentido, la segmentación característica de la educación en América Latina ha provocado que se comparen las escuelas según su dependencia administrativa y modo de financiamiento. En el caso de Chile, a los rasgos ya descritos de su sistema educacional, se agrega el componente de grupo socioeconómico que atienden las diferentes escuelas.

Dichas variables han sido muy estudiadas y asociadas al rendimiento del estudiante; no obstante, los resultados han sido heterogéneos. Por ejemplo, un estudio en España afirma que las diferencias de puntuaciones que se observan a favor de los centros privados no las explica la titularidad del centro, sino las variables que se refieren a los usuarios (Calero \& Escardibul, 2007). También en España, otra investigación reveló que las escuelas secundarias públicas son más eficientes que las privadas subvencionadas (Mancebón-Torrubia et al., 2010). En Chile, algunos estudios han concluido que las escuelas particulares, con o sin subvención del Estado, cuyo financiamiento incluye recursos de los padres y atienden a grupos socioeconómicos medios o más altos, son las que obtienen en promedio los mejores resultados de aprendizaje en lenguaje y en matemáticas (MINEDUC, 2002; Mizala \& Romaguera, 2000). Otro trabajo reciente en Chile —un metanálisis sobre 17 investigaciones versadas en este tema- sostiene que hay una diferencia estadísticamente significativa a favor de las escuelas particulares subvencionadas (Drago \& Paredes, 2011).

En América Latina, los estudios desde la perspectiva de la investigación sobre la eficacia escolar han concentrado su interés en determinar los factores que se asocian al rendimiento escolar, entre ellos el análisis de los efectos escolares (Murillo, 2007; Zorrilla, 2008). Al respecto, ya se ha buscado estimar la magnitud de efecto escolar en el rendimiento en matemáticas y lectura de estudiantes latinoamericanos de tercer y sexto grado de primaria, y compararlo con el efecto del nivel cultural y socioeconómico de las familias. Para ello se usaron datos del Segundo Estudio Comparativo y Explicativo (SERCE) de la OREALC/UnESCO y metodología de modelos multinivel (Murillo \& Román, 2011).

Entre los hallazgos se confirmó que el efecto escolar neto para el conjunto de América Latina se encuentra entre 17.6 y 22.3\%, con diferencias entre los países, y el efecto del nivel cultural y socioeconómico entre el 10.8 y el 14.0\%. Por ejemplo, en Chile, para lectura en ambos grados, el "efecto cuna" (nivel cultural y socioeconómico de las familias) es superior al efecto escuela y el aporte de esta al rendimiento escolar tiene efectos inferiores al 15\%, en promedio (Murillo \& Román, 2011).

Otros factores de contexto relevados por la literatura son las características socioeconómicas de los estudiantes, como la educación y la renta de los padres 
(Anderson et al., 2010; Milford et al., 2010; OECD, 2009). Asimismo, el número de libros en el hogar, asociándose más cantidad de estos a un mejor desempeño académico - sobre todo cuando este proceso ocurre en la vida temprana del niño- (mineduc, 2009, 2010), y a un mejor desarrollo cognitivo y del lenguaje (Tomopoulos et al., 2006).

La investigación ha mostrado que el ambiente familiar es de significativa importancia, pues representa la primera instancia formativa de un individuo (Guevara, 1996). Mead, citado por Berger \& Luckmann (2003: p. 164), afirma que "el individuo nace dentro de una estructura social objetiva en la que encuentra a otros significantes cargados de su socialización y que le son impuestos". Dicho proceso es conocido como socialización primaria. Estos otros significantes que mediatizan el mundo para el niño, también lo modifican durante tal proceso, seleccionando rasgos de la realidad, según la posición que aquellos ocupan en la estructura social (Berger \& Luckamnn, 2003). Esto deviene en la gestación de diversas "estructuras estructuradas, principios generadores de prácticas distintas y distintivas", denominados habitus (Bourdieu, 1998: p. 33). Del mismo modo, los habitus son "estructuras estructurantes, esquemas clasificatorios, principios de clasificación, principios de visión y división, de gustos” y prácticas diferentes (Bourdieu, 1998: pp. 33-34). Posterior a la socialización primaria, la socialización secundaria corresponde a la "internalización de submundos institucionales o basados en instituciones" (Berger \& Luckamnn, 2003: p. 172), cuyo alcance y tipo se definen por la división del trabajo y la correspondiente distribución social del conocimiento.

En este contexto, las expectativas educacionales de la familia, específicamente de los padres y el desarrollo de la autoestima del niño, cumplen un rol relevante (Kirk et al., 2011; Lam et al., 2013; Phillipson, 2007; Räty, 2006; Villiger et al., 2012). Sobre ello, Maslow (1943), en su jerarquía de las necesidades humanas, señala que los seres humanos tenemos necesidad de aprecio de uno mismo y de otras personas. En este sentido, una investigación hecha con una muestra de adolescentes en Rumania, tuvo como objetivo determinar los estilos parentales para identificar el nivel de autoestima del estudiante y su efecto en el rendimiento. El estudio confirmó una relación entre el estilo autoritario y una autoestima positiva, y la autoestima positiva con la mejora de los resultados escolares (Mogonea \& Mogonea, 2014).

Desde la teoría cognitiva social se ha planteado que los niños reciben la influencia de modelos consistentes a lo largo de su vida, como los padres; cuando estos tienen altas expectativas educativas de sus hijos les transmiten tales aspiraciones y la creencia de que son alcanzables (Bandura et al., 1996). La investigación indica que una participación temprana de los padres en la escuela, en especial en el nivel preescolar y jardín infantil, contribuye al desarrollo de habilidades de 
preparación escolar en los hijos (Davison et al., 2004), lo que influye de modo importante en su desarrollo académico futuro (Mendez, 2010).

Además, cuando la participación de los padres ocurre de manera temprana, con calidad y se combina con altas expectativas, tiene efectos significativos positivos en el logro académico de los niños(as) (Englund et al., 2004). Otro estudio encontró que la participación de los padres en la enseñanza secundaria, como el apoyo en las tareas escolares, no tiene el mismo efecto positivo en los resultados de aprendizaje, que en los primeros grados (Hill \& Tyson, 2009). Con relación a la forma en cómo los padres perciben y formulan expectativas y aspiraciones sobre los resultados educativos y ocupacionales de sus hijos, otra investigación concluyó que ellas influyen positiva y notoriamente en los grupos más educados (Vryonides \& Gouvias, 2012).

En la misma perspectiva, un estudio de Froiland et al. (2012) contribuye a comprender mejor los efectos del entorno familiar en los resultados educativos. Utilizando una muestra representativa a nivel nacional de estudiantes de jardín infantil y sus padres en los Estados Unidos, llegó a la conclusión de que las expectativas tempranas de los padres para el logro educativo postsecundario tienen un efecto más fuerte sobre el logro en octavo grado, que la participación paternal en casa, como, por ejemplo, en la tareas de los niños. Además, las expectativas de los padres influencian las expectativas de los estudiantes y predicen significativamente el logro académico en octavo grado (Bravo et al., 2015).

Otras investigaciones han arrojado como determinantes variables a nivel individual. Tal es el caso de las características de la motivación (Wormington $e t$ al., 2012), la edad (Ma, 2005), el efecto de los pares (efecto peer) (Carmichael \& Taylor, 2005), el género — especialmente en el desempeño en matemática(Chen et al., 2013), la autopercepción de logro y la automotivación hacia los objetivos (Cheng \& Lam, 2013), el estilo cognitivo (Hederich, 2007; López et al., 2011), y la orientación a la tarea (Hirvonen et al., 2012).

Una variable individual muy importante en educación es la autoeficacia, considerada como una variable psicológica y uno de los elementos centrales de la teoría cognitiva social (Bandura, 1977). La autoeficacia se refiere a la creencia de una persona, en este caso el estudiante, para organizar y ejecutar las estrategias que considera necesarias para conseguir determinados logros en una tarea dada, o enfrentar el cambio en la sociedad y el que a futuro espera en un puesto de trabajo (Bandura, 1986). Investigaciones recientes han demostrado la influencia de la autoeficacia en el interés que demuestra el estudiante por la tarea, la motivación y la persistencia en los objetivos (Moreira et al., 2013).

Diversos estudios empíricos han confirmado que la autoeficacia muestra un mayor poder predictivo sobre el rendimiento académico que otras variables 
cognitivas (Bandura, 1982). Además, se ha enfatizado en el rol de las expectativas de eficacia, referidas a la creencia que una persona posee sobre su capacidad de realizar comportamientos, señalando que las expectativas de eficacia pueden variar en magnitud (grado de dificultad de una tarea determinada), generalidad (se crean expectativas generales), e intensidad (facilidad o dificultad frente a una experiencia) (Bandura, 1997).

La investigación en Chile corrobora la evidencia empírica internacional y ha comprobado la alta correlación entre el índice socioeconómico y cultural y el rendimiento académico (Villalobos \& Valenzuela, 2012).

En evaluaciones internacionales, Chile participa hace más de una década en el Programme for International Student Assessment (PISA), un proyecto de la Organization for Economic Co-operation and Development (OECD). El principal objetivo de dicho programa es evaluar las competencias del estudiantado de 15 años en tres grandes áreas de aprendizaje: lenguaje, matemática y ciencias (OECD, 2007). Los resultados de las pruebas indican que Chile se encuentra por debajo del promedio (OECD, 2012). No obstante, cuando se compara con los países de América del Sur, se observa que Chile se encuentra entre los mejores de la región (Cariola et al., 2009; Salvo et al., 2012). Estos resultados son similares a los que Chile obtiene en el Trends in International Mathematics and Science Study (TiMss), programa desarrollado por la Asociación Internacional para la Evaluación del Rendimiento Educativo (IEA), el cual permite medir a estudiantes de cuarto y octavo grado básicos.

Así, Chile aparece como el país con la mayor segregación socioeconómica en el sistema educativo (OECD, 2012). Lo anterior se perpetúa en las propias aulas, espacio desde el cual han sido expresadas fuertemente en el último tiempo las demandas estudiantiles (Bellei et al., 2004). Estudios nacionales sostienen que el sistema escolar chileno se caracteriza por una temprana y alta condicionalidad del nivel socioeconómico en el desempeño académico y un bajo nivel de movilidad ascendente (Valenzuela et al., 2008; Villalobos \& Valenzuela, 2012). De esta manera, los desempeños de los estudiantes no evidencian una mejora sostenida en el tiempo (Gershberg et al., 2012).

Los factores relevados por la investigación educativa interactúan y agrupan a sujetos con características comunes en cuanto a nivel de desempeño y particularidades individuales y familiares. Se crean así perfiles de estudiantes. Esto permite contar con una información básica que describe a grupos determinados, lo que ha llevado a observar la forma en cómo interaccionan las variables en estos grupos y facilitar el diseño de medidas contextualizadas para la mejora educativa (Ma, 2005).

El presente estudio busca identificar y caracterizar perfiles de estudiantes chilenos en función del rendimiento académico en la prueba Simce 2011 de 
Historia, Geografía y Ciencias Sociales. Ello para responder una pregunta de investigación: ¿qué factores constituyen los perfiles de desempeño de los estudiantes chilenos de octavo grado básico en la prueba Simce 2011 de Historia, Geografía y Ciencias Sociales?

\section{Metodología}

Esta investigación es de tipo cuantitativo, es decir, que se estructura en fases lógicamente secuenciales, según un planteamiento deductivo, que se mueve en el contexto de la corroboración empírica de la teoría formulada previamente (Corbeta, 2007). Sigue un enfoque correlacional predictivo y como técnica de análisis estadístico utiliza el árbol de clasificación y regresión (CART) (Breiman et al., 1984).

Participantes. Los participantes del estudio se encuentran asociados a la base de datos del Simce para el dominio Historia, Geografía y Ciencias Sociales, con una población de 221154 estudiantes de octavo grado (media 13.65 años/DS $0.74)$ que rindieron la prueba los días 24 y 25 de octubre de 2011. La distribución de los estudiantes corresponde a 5863 establecimientos, que representan al 99\% de los que imparten el octavo grado básico en el país. La asistencia de los estudiantes los días en que se aplicaron las pruebas Simce fue de 95\%, y la asistencia promedio a lo largo del año escolar 2011 fue de 92\% (MINEDUC, 2011).

Base de datos del Simce del Ministerio de Educación de Chile. Es un sistema de evaluación censal fundado en 1988, administrado por la Agencia de la Calidad de la Educación. Los datos del Simce son públicos para investigación y se obtuvieron previo acuerdo de confidencialidad (MINEDUC, 2011).

Las pruebas Simce evalúan el logro de los "Objetivos Fundamentales" y los "Contenidos Mínimos Obligatorios del Marco Curricular" vigente en diferentes subsectores del aprendizaje. Además, el Simce recoge información de profesores, estudiantes y padres y apoderados, a través de cuestionarios de calidad y contexto de la educación chilena.

La base de datos fue etiquetada y codificada para todas las variables, indicando el cuestionario, pregunta e ítem. Fueron identificadas las categorías de vacías y de doble marca. Los ítems que fueron respondidos manualmente, como fechas y edades, fueron validados. Algunas preguntas como el nivel de estudios del padre o la madre se agruparon para su mejor comprensión.

La validez y confiabilidad del Simce se basa en el modelo de medición de la teoría de respuesta al ítem (TRI), el cual permite la comparabilidad de los pun- 
tajes a través del tiempo, y posibilita comparar con precisión el rendimiento de diferentes cohortes, aun utilizando pruebas distintas, debido a que utiliza el procedimiento de equiparación o equating (MINEDUC, 2012).

Prueba de Historia, Geografía y Ciencias Sociales. Los resultados reportados de esta prueba se expresan en puntajes por estudiante y corresponden a la variable dependiente. Se trata de una prueba estandarizada bianual para este dominio, que incorpora la evaluación de habilidades como analizar e interpretar diversas fuentes referidas a procesos históricos y fenómenos geográficos, políticos y económicos.

Cuestionarios de contexto. Otros instrumentos utilizados fueron los cuestionarios de contexto, a saber:

- Cuestionario del Estudiante. Que se compone de treinta preguntas y alcanza 143 ítems de respuesta: de aquí se obtuvieron variables como género, edad, percepción de autoeficacia, percepción del clima de aula, y percepción sobre las expectativas educacionales del estudiante, entre otras.

- Cuestionario de Padres. Conformado por 31 preguntas y 119 ítems de respuesta, de este se obtuvieron variables sobre el nivel cultural de los padres (máximo nivel de escolaridad del padre y la madre, cantidad de libros en el hogar), nivel socioeconómico de la familia (rangos de ingresos en el hogar, posesión de artefactos tecnológicos, etc.), expectativas educacionales sobre sus hijos e hijas, años de repitencia de sus hijos(as), y años de educación preescolar de sus hijos(as), entre otras.

- Cuestionario de Docentes de Historia, Geografía y Educación Cívica. Integrado de 37 preguntas y 193 ítems de respuesta. De este se obtuvieron variables como el avance curricular, expectativas educacionales sobre los estudiantes, percepción de violencia en la escuela, entre otras.

La información de establecimientos educacionales incluye región, provincia, comuna, ruralidad, dependencia o tipo de establecimiento, y grupo de rendimiento.

Considerando lo anterior, la matriz de datos que se utilizó para el análisis CART fue de 221154 individuos y 53 variables e índices de contexto de estudiantes, padres y profesores.

Desempeño escolar en la prueba de Historia, Geografía y Ciencias Sociales

Acerca del puntaje en la prueba, este presentó una media de 259.5 puntos y la desviación estándar fue de 48.2, con puntajes mínimo y máximo de 146 
y 406, respectivamente. El 75\% de los estudiantes de octavo básico no superaron los 294.6 puntos. El 20\% obtuvo puntajes superiores a 300 y el $2.5 \%$ de los estudiantes rebasaron los 355. Los resultados en las últimas evaluaciones evidencian una mejora significativa de ocho puntos comparado esto con la evaluación Simce 2009 (MINEDUC, 2011).

\section{Procedimiento}

\section{Construcción de índices}

En total se construyeron 29 índices referidos al contexto del estudiante, padres y profesores. Para operacionalizar el estudio, solo se describe el que fue relevante para el análisis y que se denominó indice de autoeficacia (IA). Este hace referencia a la percepción que el estudiante tiene de su capacidad de estudio, esfuerzo, motivación, y además constata el cumplimiento de algunas labores escolares como el cumplimiento de tareas y tomar apuntes en clases. Las respuestas se expresan en términos del grado de acuerdo o desacuerdo.

El IA se construyó sumando los nueve ítems de la pregunta 6 del Cuestionario del Estudiante, cuya escala es de tipo Likert, mismo que varía de 1 a 4 (muy en desacuerdo a muy de acuerdo). La tabla 1 muestra la pregunta y sus respectivos ítems. No se reporta nivel de confiabilidad porque, como se indica, el índice se construyó sumando los ítems que lo componen, tal como se hizo en Yoo \& Donthu (2001) y en Bravo et al. (2015). Este cálculo no es afectado por el tipo de escala (si va de 1 a 4 o de 1 a 5), y el rango de variabilidad es mayor, pero el sentido es el mismo.

Como se puede observar, algunos de los ítems del IA parecen estar más directamente relacionados que otros con el concepto de autoeficacia. En particular, es importante anotar las diferencias entre los ítems 1-4, directamente referidos a las percepciones del estudiante sobre su capacidad y su desempeño, y los ítems 5-9, más relacionados con aspectos de hechos, gustos y orientación motivacional. La literatura evidencia que la autoeficacia es una variable motivacional cuya principal influencia sobre el desempeño académico se observa en las conductas de persistencia, organización y ejecución de estrategias consideradas necesarias para alcanzar logros en una tarea, además de resistencia a la frustración (Bandura, 1986, 1997), aspectos descritos en los ítems 5-9. Por estas razones el índice de autoeficacia mantiene una unidad teórica.

Como se generaron muchos índices, para poder compararlos fueron estandarizados (media 0 y DS de 1). En particular, valores negativos del IA significan 
que la percepción de autoeficacia del estudiante está en el promedio, y valores positivos sobre el promedio. El IA varía entre -4.01 y 2.19 .

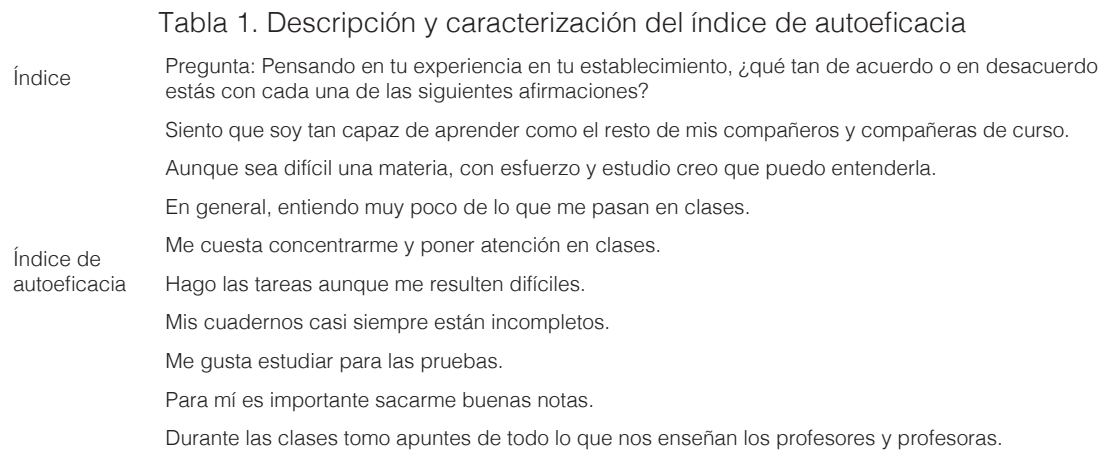

Fuente: Cuestionario del Estudiante, Simce 2011. Recuperado de http://www.agenciaeducacion.cl/biblioteca-digital/ cuestionarios-alumnos/

\section{Análisis}

Los perfiles de estudiantes se obtuvieron mediante el análisis multivariado CART. Este corresponde a un enfoque exploratorio de extracción de relaciones de dependencia jerárquica entre una variable respuesta o dependiente y un conjunto de variables influyentes (Breiman et al., 1984). Se considera como variable dependiente el desempeño académico en la prueba de Historia, Geografía y Ciencias Sociales. De acuerdo a Ma (2005), quien fue pionero en la aplicación de esta técnica en la investigación educativa, señala que el CART lleva a cabo la separación en grupos sucesivos con base en un criterio estadístico. Comenzando en este caso por la población completa (nodo raíz), cada variable explicativa se examina de modo que divide a los estudiantes en dos grupos (nodos hijos). El CART da una medida llamada impureza para guiar la división. Ella mide el grado en que los estudiantes en un nodo varían en la medida del resultado. Una impureza menor indica un resultado más homogéneo para un nodo, y su disminución se puede calcular al comparar la del nodo raíz con la suma de las impurezas de su nodo hijo. La variable explicativa que rinde la disminución más grande en impureza se selecciona para hacer la primera división. Así, los nodos hijos resultantes son notoriamente distintos en la medida del resultado. Luego, cada nodo se divide de nuevo siguiendo el mismo procedimiento. Según el proceso continúa, los estudiantes son clasificados en nodos cada vez más pequeños. La similitud en la medida del resultado dentro de cada nodo aumenta, mientras que la diferencia de esto mismo entre los nodos también crece. Los 
nodos que han llegado a su límite de división son llamados terminales. Así, son seleccionadas las variables que explican el rendimiento buscando la mayor separabilidad entre grupos.

Esta técnica posee muchas ventajas respecto de las más tradicionales utilizadas en el área de la educación. Cuando se usa el CART, la identificación correcta y la modelación de los efectos interactivos no son necesarios porque aquel genera grupos mutuamente excluyentes que proveen un cuadro directo y automático de los efectos interactivos de medidas explicativas importantes. Como no desarrolla un modelo estadístico, no produce ecuaciones matemáticas complejas, lo que hace que los resultados sean fáciles de entender e interpretar, pues cada grupo puede describir completamente las características de los individuos en ese grupo, y cada grupo puede tener un promedio estimado de la medida del resultado (Schiattino \& Silva, 2008; Ma, 2005).

Por último, el CART es esencialmente una técnica estadística no paramétrica, por lo cual no requiere distribución específica para el análisis de los datos. Lo anterior hace del CART una técnica estadística atractiva para el análisis explicativo de los datos (Ma, 2005).

La presentación de la información se hace en un diagrama en forma de árbol invertido. Un árbol es un conjunto de nodos y arcos. El nodo raíz representa a toda la población y los demás a un subconjunto de la población. Se distinguen los nodos intermedios cuyos arcos salientes apuntan a los hijos y los terminales que significan la partición final (Schiattino \& Silva, 2008).

Para procesar la información se utilizó el software estadístico JMP ${ }^{\circledast} 10$.

\section{Resultados}

De todas las variables disponibles en la base de datos del Simce (variables del estudiante, del contexto familiar y las del contexto colegio), el análisis CART arrojó cinco que son determinantes para explicar el desempeño de los estudiantes en la prueba Simce de Historia, Geografía y Ciencias Sociales.

En la tabla 2 se muestra dichas variables, según su orden de importancia. Estos porcentajes de explicación representan una proporción del grado relativo de relevancia entre los factores seleccionados en el modelo CART, y destacan, por su contribución a la explicación de la varianza, las Expectativas educacionales de la familia. De manera conjunta estas contribuyen con un $20 \%$ de varianza explicada de la variable dependiente, lo que es educacionalmente relevante, tal como lo demuestran otros estudios en educación que han utilizado para el análisis modelos de regresión (Reyes et al., 2007; Abdullah et al., 2013). 
Tabla 2. Variables relevantes según el análisis CART para explicar los resultados Simce 2011 en la prueba de Historia, Geografía y Ciencias Sociales

\begin{tabular}{lc}
\hline Variables & \% contribución \\
\hline Expectativas educacionales de la familia & 56.0 \\
Máxima escolaridad de los padres & 23.6 \\
Dependencia de la escuela & 11.7 \\
Índice de autoeficacia (IA) del estudiante & 7.1 \\
Número de libros en el hogar & 1.6 \\
\hline
\end{tabular}

Fuente: Elaboración propia.

La variable Expectativas educacionales de la familia fue creada a partir de la pregunta 23 del Cuestionario de Padres "¿Cuál cree usted que es el nivel de educación más alto que el estudiante podrá completar en el futuro?” Las respuestas se agruparon por el CART en dos variables: a) Bajas expectativas educacionales con base en las respuestas a "No creo que complete 4o Año de Educación Media; 40 Año de Educación Media Técnico-Profesional; 40 Año de Educación Media Científico-Humanista; Una carrera en un Instituto Profesional o Centro de Formación Técnica”; y b) Altas expectativas educacionales con base en las respuestas a "Una carrera en la Universidad y Estudios de postgrado". Se consideraron las respuestas entregadas por personas que declaraban una relación de parentesco con el estudiante, de acuerdo a la pregunta 3 del Cuestionario de Padres: “¿Cuál es su relación de parentesco con el estudiante? (padre, madre, tío, abuelo, hermano, madrastra, padrastro, otro pariente, otro no pariente)". El $80.2 \%$ declaró ser la madre; un $13.5 \%$, el padre; un 6\% declaró otro parentesco; y solo el $0.3 \%$ declaró ser otro no pariente.

La variable Máxima escolaridad de los padres fue elaborada a partir de las preguntas 8 y 9 del Cuestionario de Padres, respectivamente: “¿Hasta qué nivel educacional llegó el Padre (o Padrastro) del estudiante?” y “¿Hasta qué nivel educacional llegó la Madre (o Madrastra) del estudiante?” Las respuestas fueron promediadas y agrupadas.

La variable Dependencia de la escuela o tipo de establecimiento educacional fue elaborada con la información extraída de la ficha de identificación en los cuestionarios. Es una variable politómica compuesta de tres categorías con base en los criterios de tipo de financiamiento y administración: establecimiento público bajo administración municipal, establecimiento particular subvencionado por el Estado con administración privada, y establecimiento particular pagado y administrado por privados.

Indice de autoeficacia del estudiante. Como ya fue señalado, se construyó a partir de la pregunta 6 del Cuestionario del Estudiante y se refiere a la percepción que el estudiante tiene de su capacidad de estudio, esfuerzo y motivación. 
Además, constata el cumplimiento de algunas labores escolares como la realización de tareas y tomar apuntes en clases.

Número de libros en el hogar. Esta variable se elaboró con la pregunta 7 del Cuestionario de Padres: “¿Cuántos libros hay en el hogar del estudiante? No incluya textos escolares, diarios o revistas", y las respuestas: "Ninguno; Menos de 10; Entre 10 y 50; Entre 51 y 100, y Más de 100”. Las respuestas fueron promediadas y agrupadas.

La figura 1 muestra el análisis CART de desempeño en la prueba de Historia, Geografía y Ciencias Sociales.

Figura 1. Árbol de regresión (CART) de desempeño de estudiantes de octavo grado en la prueba Simce 2011 de Historia, Geografía y Ciencias Sociales

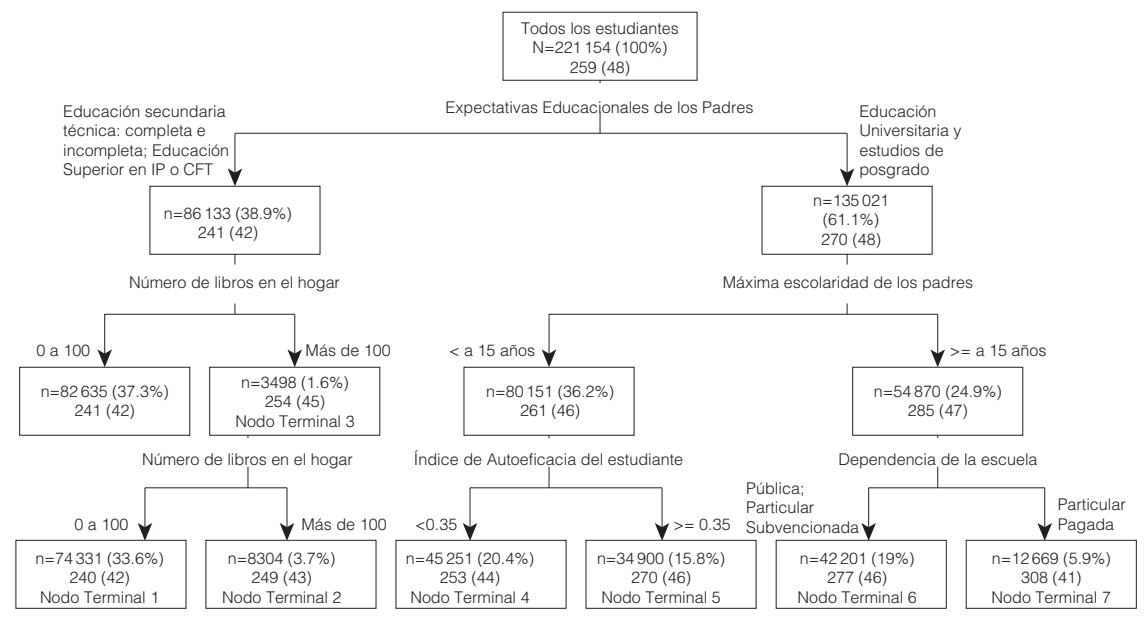

Nota: Cada nodo está asociado a la característica de la variable. Dentro del nodo se expresa el número de estudiantes y su porcentaje entre paréntesis; luego se señala el puntaje promedio en la prueba, con la desviación estándar entre paréntesis. Por último, se identifican los nodos terminales.

Fuente: Elaboración propia.

El análisis CART identificó siete nodos terminales que agrupan a los estudiantes de acuerdo a su nivel de rendimiento.

El nodo raíz, ubicado en la parte superior de la figura 1, agrupa a toda la población, con 221154 estudiantes que obtuvieron en promedio 259.5 puntos en la prueba. El nodo raíz fue dividido por la variable Expectativas educacionales de la familia. A partir de aquí, hay dos nodos hijos.

El nodo hijo de la izquierda, identificado con las expresiones "Educación secundaria técnica: completa e incompleta; Educación Superior en IP o CFT", se asocia con baja expectativa educacional. En efecto, los padres esperan que 
su hijo(a) solo complete los doce ańos de escolaridad obligatoria o asista, posteriormente, a un instituto técnico profesional (IP) o un Centro de Formación Técnica (CFT). Este nodo tiene 86133 estudiantes que representan el 39\% de la población. Fue dividido por la variable Número de libros en el hogar, lo que dio origen a los grupos o nodos terminales 1,2 y 3 .

El nodo hijo de la derecha denominado "Educación universitaria y estudios de posgrado", se encuentra asociado a altas expectativas educacionales de la familia. Estas esperan que sus hijos continúen estudios universitarios de pregrado o posgrado. Este nodo agrupa a 135021 estudiantes que representan el 61\% de la población. Puntúan en promedio 270 puntos en la prueba, puntaje que se encuentra sobre la media. Este nodo fue dividido por las variables Máxima escolaridad de los padres, Índice de autoeficacia del estudiante y Dependencia de la Escuela, lo que da origen a los nodos terminales 4, 5, 6 y 7.

La tabla 3 presenta indicadores de resumen para los siete grupos terminales sobre el rendimiento académico en la prueba. Los grupos 1-4 obtienen un rendimiento menor que el promedio. El 94\% de los estudiantes de los grupos 1-6 no superan los 300 puntos promedio. Los grupos 5, 6 y 7 consiguen los mejores rendimientos, destacando este último, que desde el percentil 40 obtiene sobre los 300 puntos.

Tabla 3. Principales indicadores por grupo, según el rendimiento académico en Historia, Geografía y Ciencias Sociales

\begin{tabular}{lccccccc}
\hline & 1 & 2 & 3 & 4 & 5 & 6 & 7 \\
\hline N & 74331 & 8304 & 3498 & 45251 & 34900 & 42201 & 12669 \\
\hline Media & 240.2 & 249.8 & 254.5 & 253.7 & 270.7 & 277.9 & 308.8 \\
D. estándar & 42.4 & 44 & 45.9 & 44.9 & 46 & 46.7 & 41.3 \\
Mediana & 236 & 247.9 & 252 & 251.9 & 271.4 & 280.6 & 311.5 \\
Mínimo & 146.7 & 146.7 & 149.3 & 146.8 & 146,8 & 147.6 & 146.7 \\
Quintil 5 & 117.4 & 181.1 & 183.1 & 183.1 & 194.2 & 197.3 & 234.3 \\
Quintil 25 & 208.1 & 216.3 & 219.8 & 219.8 & 237.1 & 244.9 & 283.9 \\
Quintil 75 & 269.2 & 280.9 & 286.4 & 285.6 & 304.2 & 311.9 & 337 \\
Quintil 95 & 315 & 325.3 & 334.5 & 330.2 & 345.6 & 352.2 & 373.4 \\
Máximo & 406.6 & 397.9 & 406.6 & 406.6 & 406.6 & 406.6 & 406.6 \\
\hline
\end{tabular}

Fuente: Elaboración propia.

A partir de los nodos terminales, se identificaron siete perfiles de los estudiantes que rindieron la prueba, tales se describen enseguida, según su orden de aparición:

1. Grupo que obtiene en promedio los desempeños más bajos en la prueba con 240 puntos, y que no supera el percentil 38 (33.6\% de la población). 
La familia de estos estudiantes señala bajas expectativas educacionales sobre su desempeño académico y declara tener entre ninguno y menos de 50 libros en el hogar.

2. Grupo que obtiene en promedio 249 puntos, y que no supera el percentil 44 (3.8\% de la población). La familia de estos estudiantes reporta bajas expectativas educacionales sobre su desempeño académico y declara poseer entre 51 y 100 libros en el hogar.

3. Grupo que obtiene en promedio 254 puntos, y que no supera el percentil 48 (1.6\% de la población). La familia de estos estudiantes indica bajas expectativas educacionales sobre su desempeño académico y declara poseer más de 100 libros en el hogar.

4. Grupo que obtiene en promedio 253 puntos, y que no supera el percentil 47 (20.5\% de la población). La familia de estos estudiantes declara altas expectativas educacionales sobre su desempeño académico y presentan menos de 15 años de escolaridad, sin superar el percentil 75. Se trata de un grupo cuyo índice de autoeficacia no rebasa el percentil 63.

5. Grupo que obtiene en promedio 270 puntos, el cual no supera el percentil 59 (15.8\% de la población). La familia de estos estudiantes declara altas expectativas educacionales sobre su desempeño académico y menos de 15 años de escolaridad, sin superar el percentil 75 . El índice de autoeficacia de este grupo supera el percentil 63.

6. Grupo que obtiene en promedio 277 puntos, que no supera el percentil 64 (19.1\% de la población). La familia de estos estudiantes declara altas expectativas educacionales sobre su desempeño académico y una escolaridad mayor a 15 ańos, con lo que supera el percentil 75. El 25.2\% de ellos estudia en establecimientos educacionales públicos y un $74.8 \%$ en colegios particulares subvencionados por el Estado.

7. Grupo que obtiene en promedio los más altos desempeños en la prueba, con 308 puntos, que corresponden al percentil 83 (5.7\% de la población). Son estudiantes con una familia que declara altas expectativas educacionales sobre su desempeño académico y una escolaridad mayor a 15 años, superando así el percentil 75. El 100\% estudia en colegios particulares privados.

\section{Discusión}

La variable Expectativas educacionales de la familia permitió establecer un primer criterio de explicación del desempeño al separar dos subgrupos de estudiantes. Respecto de los bajos resultados, se vincula con la variable Número de libros en 
el hogar, esta última con un bajo nivel de contribución (1.6\%). El subgrupo asociado con altas expectativas alberga a los nodos con rendimientos más altos y aparece relacionado con otras tres variables.

Estos resultados se condicen con evidencias empíricas que dan cuenta de la relación entre familia y desempeño (Bandura et al., 1996; Bravo et al., 2015; Englund et al., 2004; Froilan, 2012). Destacan sobre todo los dos nodos asociados a los grupos de mayor desempeño en la prueba, vinculados a las variables Expectativas educacionales de la familia y Máxima escolaridad de los padres. Este hallazgo está en línea de lo relevado por Viryonidesa \& Gouvias (2012) respecto de la influencia positiva de las expectativas familiares en los grupos más educados. Desde lo teórico, los hallazgos fortalecen la idea de que la familia se erige como un factor fundamental en los procesos de socialización primaria (Berger \& Luckmann, 2003). En la medida que este proceso se profundiza, la mayor o menor intensidad y sintonía de dicha socialización con los marcos institucionales predominantes facilita u obstaculiza la inserción exitosa de la persona en los distintos submundos institucionales. Entre estos el de la educación formal representada por la escuela (Berger \& Luckmann, 2003). Aquí, las expectativas familiares constituyen un factor — de varios - vinculado al aprendizaje de diversos saberes, los que se producen en un contexto social específico.

La variable Máxima escolaridad de los padres, que aparece asociada con las $\mathrm{Al}$ tas expectativas familiares, permite matizar la explicación de aquellos grupos que presentan resultados más altos ( $4 \mathrm{al}$ 8). En particular se distinguen dos subgrupos distanciados por una diferencia de 24 puntos, lo que es significativo. Los resultados sugieren que una mayor escolaridad de los padres explicaría mejores desempeños en la prueba.

Dicha escolaridad, como rasgo relevante de la cultura de un padre o madre, bien pudiera constituir un modelo de poderosa incidencia sobre el o los hijos. Ello estaría en línea de lo planteado por la teoría cognitiva social (Bandura et al., 1996).

Desde ciertas perspectivas sociológicas también se puede asumir que mayores niveles de escolaridad permiten una mejor inserción en ciertos espacios institucionales, como los asociados al ámbito de las profesiones. Especialmente, las más cotizadas. Para alcanzar estas metas deben desplegarse esquemas de clasificación y producción de prácticas de naturaleza social, que en este caso, forman parte del habitus de los subgrupos que ven en la educación formal un mecanismo para la obtención de su estatus y desarrollo social (Bourdieu, 2008). Dichos esquemas son transmitidos a sus descendientes a partir de la primera socialización y esto impacta en los mejores desempeños que estos últimos presentan en relación con sus pares cuyos padres no alcanzan esos niveles de escolaridad. No obstante, no es posible explicar con claridad cuáles serían 
los aspectos específicos que configuran estos esquemas o habitus para el caso de este análisis, lo que constituye otra de sus limitaciones. Creemos que sería relevante indagar en aspectos específicos asociados a la variable escolaridad de los padres, como la transmisión de hábitos de estudio, materias escolares consideradas más relevantes por los padres o el desarrollo de una mayor motivación modelada con los ejemplos de los progenitores y su incidencia en el desempeño escolar en general.

La variable Dependencia de la escuela permitió desagregar los últimos dos grupos (nodos terminales 6 y 7), que aparecen separados por una brecha de 31 puntos, lo que resulta importante. Tomando en cuenta que las otras variables altamente incidentes, según el CART, son las mismas, entonces cabe preguntarse por el impacto de la educación escolar (efecto escuela) en dichos rendimientos. A la luz de este hallazgo se cree necesario profundizar en estudios que permitan observar con mayor precisión y mayor nivel de significación estadística el efecto del colegio en los desempeños.

Resulta interesante la relevancia que el CART le asigna a la variable Número de libros en el hogar, que aparece asociada a las expectativas familiares. Ello porque permite desagregar los grupos 1,2 y 3 , vinculados a los más bajos resultados en la prueba. Es decir, a diferencia de los estudios que destacan positivamente mayores logros asociados a una mayor cantidad de libros (MINEDUC, 2009, 2010), lo que encuentra esta investigación es la asociación entre bajos resultados y menor cantidad de libros disponibles. A lo anterior se añade que el CART no recuperó esta variable como relevante para explicar los buenos resultados. A partir de este hallazgo sería recomendable emprender futuros estudios que indaguen en la jerarquización de los factores que inciden en los desempeños escolares. En especial ponderar la importancia de la cantidad de libros en el hogar como variable explicativa de los desempeños. Por otra parte, aun cuando el Número de libros en el hogar tiene una baja contribución de acuerdo al CART —alrededor de 1.6\%—, las diferencias en los puntajes de estos grupos tienen cierta importancia, sobre todo al contrastar los valores extremos (grupos 1 y 3). Esto sugiere varias cosas. Por ejemplo, que la escasa cantidad de libros responde a las bajas expectativas de la familia con relación a los hijos. O bien, que las familias no lo consideran como un factor no relevante para el desempeño exitoso. Luego, eso se transforma en un factor determinante para el éxito o el fracaso en pruebas como la que se analiza en este estudio, cuestión que podría generalizarse al desempeño escolar.

Desde una mirada más focalizada en esta variable, distintas investigaciones confirman la relación entre aprendizaje y buenos logros escolares cuando hay un mayor acceso a la información y la cultura. No obstante, según los resultados de esta investigación no es posible sostener que más libros en el hogar ga- 
rantice que se asocian a los saberes de la escuela o que son leídos y consultados por los estudiantes. El CART no lo recogió para explicar los mejores resultados. Además, la base de datos a la que se accedió no permitió profundizar en este punto, lo cual representa una limitación de este trabajo. Sería interesante indagar más en la relación entre expectativas familiares respecto a la educación formal con la cantidad y calidad de la cultura disponible a través de libros y otros dispositivos portadores de saber y cultura. Se considera aún más relevante en un momento epocal en el que el acceso a la cultura se supone más abierto y expedito en función del crecimiento explosivo de las tecnologías de la información y la comunicación.

La variable Índice de autoeficacia, relevada por el CART en asociación con Expectativas familiares y el Nivel de escolaridad de los padres permite desagregar los grupos 4 y 5 que presentan una brecha de 17 puntos, un aspecto importante. Diversos estudios destacan la trascendencia de la disposición del propio sujeto para lograr desempeños exitosos en el entorno escolar (Bandura, 1977, 1982, 1986, 1997; Moreira et al., 2013). Con todo, aun cuando el factor individual — representado aquí por el IA — puede incidir para establecer diferencias, al menos para este estudio aparece subordinado a factores de naturaleza social, como las expectativas educacionales de la familia y el nivel de escolaridad de los padres. Esto sugiere un alcance limitado de este factor cuando se intenta explicar los desempeños desde una perspectiva más sistémica y contradice algunas perspectivas que sostienen que la sola disposición individual permite vencer los desafíos planteados por el mundo escolar. Es decir, según los hallazgos de este estudio, la existencia de casos donde la autoeficacia sea más relevante que otros factores serían excepciones y no la regla.

Por último, la variable Dependencia de la escuela explica la diferencia entre los grupos 6 y 7 , que son los de más alto puntaje promedio, pero que aparecen separados por una importante brecha de 31 puntos en favor de los establecimientos pagados. Numerosos estudios confirman diferencias ostensibles en los resultados de pruebas como las que se analiza aquí (Drago \& Paredes, 2011; Mineduc, 2002; Mizala \& Romaguera, 2000). Una parte de estos estudios interpreta que los mejores resultados de los colegios pagados se deben a que sus estudiantes detentan mayores niveles de capital cultural y económico (Calero $\&$ Escardibul, 2007). Esto supone una mayor familiaridad de estos grupos con patrones y esquemas socioculturales que se condicen con aquellos de los ámbitos institucionales, como el de la educación formal. A pesar de todo, creemos que todavía no existe suficiente investigación que dé cuenta del efecto ejercido específicamente por el colegio y la labor del profesorado, según nivel de dependencia. De este estudio solo pueden emanar sugerencias en ese sentido, pues tampoco permite evidenciar esa incidencia. 


\section{Conclusiones}

Esta investigación ha pretendido explorar los factores que explican el desempeño escolar, a partir de los resultados que tuvieron los estudiantes de octavo grado básico en la prueba de Historia, Geografía y Ciencias Sociales, del Sistema de Medición de la Calidad de la Educación de Chile. Así es como se identificaron y caracterizaron perfiles de estudiantes en función de su desempeńo y otras variables de contexto.

Es necesario señalar que las variables con las que se realizó el análisis, son las que están disponibles en la base de datos y no variables que los investigadores hayan decidido medir, lo que representa una limitación del estudio. De igual forma, cabe aclarar que el estudio explica el $20 \%$ de la varianza, lo que, si bien es significativo en estudios educativos, requiere de más investigaciones que permitan fortalecer estos resultados.

En conclusión se puede afirmar que las Expectativas educacionales de la familia fueron ser la variable de más relevancia para explicar el desempeño. Esto sugiere que las expectativas educacionales que los padres tienen de sus hijos constituyen un potencial socio-psicológico de primer orden. Especialmente, bajas expectativas se asocian con mayor claridad a bajos desempeños. En cuanto a los mejores desempeños, las expectativas se asociaron con otras dos variables de tipo socioeducativo: nivel de escolaridad de los padres y dependencia del establecimiento. Solo destaca una variable asociada a un factor de logro individual, el índice de autoeficacia. Esto reafirma la incidencia del entorno social para el logro de buenos desempeños. ¿¿De qué forma específica las expectativas de los padres impactan los desempeños escolares de sus hijos?, es algo que debiera ser estudiado más profundamente. Sobre todo investigar en las diversas interacciones del estudiante con su familia y otros elementos del entorno social en el que se desenvuelve. Respecto de lo anterior, también debería estudiarse en los diversos procesos que permiten dar cuenta de la calidad de la enseñanza, según los niveles de dependencia del establecimiento. Finalmente, en cuanto a las diferencias de capital cultural y económico que se plasman en un entorno escolar que promueve la segregación, como el caso chileno, y que han sido ampliamente divulgadas por la literatura, pueden contener elementos relevantes atribuibles a la acción de los colegios.

\section{Referencias}

Abdullah, A., Doucouliagos, H. \& Manning, E. (2013). Does Education Reduce income Inequality? A Meta-regression Analysis. Journal of Economic Surveys, 00(0), 1-16. doi: 10.1111 /joes. 12056 
Anderson, J., Chui, M. H., \& Yore, L. (2010). First Cycle of PISA (2000-2006). International Perspectives on Successes and Challengers: Research and Policy Directions. International Journal of Science and Mathematics Education, 8(3), 593-609.

Bandura, A. (1997). Self-efficacy: The Exercise of Control. Nueva York: N.H. Freeman.

Bandura, A. (1986). Social Foundations of Thought and Action: A Social Cognitive Theory. Englewood Cliffs, NJ: Prentice- Hall, Inc.

Bandura, A. (1982). Self-efficacy Mechanism in Human Agency. American Psychologist, 37 (2), 122-147.

Bandura, A. (1977). Social Learning Theory. Englewood Cliffs, NJ: Prentice- Hall, Inc.

Bandura, A., Barbaranelli, C., Caprara, G. V., \& Pastorelli, C. (1996). Multifaceted Impact of Self-efficacy Beliefs on Academic Functioning. Child Development, (67), 1206-1222.

Bellei, C., Poblete, X., Sepúlveda, P., Orellana, V. \& Abarca, G. (2013). Situación educativa de América Latina y el Caribe: Hacia la educación para todos 2015. Centro de Investigación Avanzada en Educación, (7), Recuperado de http://www.ciae.uchile.cl/index. php?page=view_publicacion $\&$ id_publicaciones $=357$

Bellei, C., Muñoz, G., Pérez, L. M. \& Raczynski, D. (2004). Escuelas Efectivas en Sectores de Pobreza: ¿Quién dijo que no se puede? UNICEF/Gobierno de Chile. Recuperado de http://www .unicef.cl/centrodoc/escuelas_efectivas/

Berger, P. \& Luckmann, T. (2003). La construcción social de la realidad. Buenos Aires: Amorrortu.

Blanco, E. (2009). Eficacia escolar y desigualdad: aportes para la política educativa. Perfiles Latinoamericanos, 17(34), 51-85.

Bourdieu, P. (2008). Capital cultural, escuela y espacio social. Buenos Aires: Siglo Veintiuno Editores.

Bravo, M., Salvo, S. \& Muñoz, C. (2015). Profiles of Chilean Students According to Academic Performance in Mathematics: An Exploratory Study Using Classification Trees and Random Forests. Studies in Educational Evaluation, (44), 50-59.

Breiman, L., Friedman, J., Olshen, R. A. \& Stone, C. (1984). Classification and Regression Trees. Belmont, CA: Wadsworth.

Calero, J. \& Escardibul, J. (2007). Evaluación de servicios educativos: El rendimiento en los centros públicos y privados en PISA 2003. Hacienda Pública Españolal Revista de Economía Pública, 183(4), 33-66. 
Cariola, L., Cares, G. \& Lagos, E. (2009). ¿Qué nos dice PISA sobre la educación de los jóvenes en Chile? Nuevos análisis y perspectivas sobre los resultados en PISA 2006. Santiago de Chile: Ministerio de Educación, Unidad de Currículum y Evaluación.

Carmichael, C. \& Taylor, J. (2005). Analysis of Student Beliefs in a Tertiary Preparatory Mathematics Course. International Journal of Mathematical Education in Science and Technology, 36(7), 713-719.

Corbetta, P. (2007). Métodos y técnicas de investigación social. Madrid: Prentice Hall.

Cox, C. \& Lemaitre, M. J. (1999). Market and State Principles of Reform in Chilean Education. En Perry, G. \& Leipziger, D. M. (Eds.). Chile: Recent Policy Lessons and Emerging Challenges (pp. 149-188). Washington: wвi Development Studies, World Bank.

Chen, H., Chen, M., Lee, Y., Chen, H. \& Keith, T. (2013). Gender Reality Regarding Mathematic Outcomes of Students Aged 9 to 15 Years in Taiwan. Learning and Individual Differences, (26), 55-63.

Cheng, R. \& Lam, S. (2013). The Interaction between Social Goals and Self-construal on Achievement Motivation. Contemporary Educational Psychology, 38(2), 136-148.

Chumacero, R., Gómez, D. \& Paredes, R. (2011). I Would Walk 500 Miles (if it Paid): Vouchers and School Choice in Chile. Economics of Education Review, 30(5), 1103-1114.

Davison, M., Seo, Y., Davenport, E., Butterbaugh, D. \& Davison, L. (2004). When do Children Fall Behind? What Can Be Done? Phi Delta Kappan, 85(10), 752-761.

Drago, J. \& Paredes, R. (2011). Meta-Análisis sobre brecha de la Calidad de la Educación en Chile. Revista Review CEPAL, (104), 167-180.

Englund, M., Luckner, A., Whaley, G. \& Egeland, B. (2004). Children's Achievement in Early Elementary School: Longitudinal Effects of Parental Involvement, Expectations, and Quality of Assistance. Journal of Educational Psychology, 96(4), 723-730.

Friedman, M. (1955). The Role of Government in Education. En Solo, R. A. (Ed.). Economics and the Public Interest (pp. 127-134). New Brunswick, NJ: Rutgers University Press.

Froiland, J., Peterson, A. \& Davison, M. (2012). The Long-Term Effects of Early Parent Involvement and Parent Expectation in the USA. School Psychology International, 34(1), 33-50.

García, C. \& Paredes, R. (2010). Reducing the Educational Gap in Chile: Good Results in Vulnerable Groups. Journal of Development Studies, 46(3). Recuperado de http://www 
.ceppe.cl/articulos-politicas-educativas/281-reducing-the-educacional-gap-good-results-en -vulnerable-groups-garcia-palomer-paredes

Gershberg, A., González, P. \& Meade, B. (2012). Understanding and Improving Accountability in Education: A Conceptual Framework and Guideposts from Three Decentralization Reform Experiences in Latin America. World Development, 40(5), 1024-1041.

Guevara, N. (1996). La relación familia-escuela. Educación 2001, (9), 6-13.

Hederich, C. (2007). Estilo cognitivo en la dimensión de Independencia. Dependencia de Campo. Influencias culturales e implicaciones para la educación. Tesis doctoral de la Universidad Autónoma de Barcelona.

Hill, N. \& Tyson, D. (2009). Parental Involvement in Middle School: A Meta-analytic Assessment of the Strategies that Promote Achievement. Developmental Psychology, (45), 740-763.

Hirvonen, R., Tolvanen, A., Aunola, K. \& Nurmi, J. (2012). The Developmental Dynamics of Task-avoidant behavior and Math Performance in Kindergarten and Elementary School. Learning and Individual Differences, 22(6), 715-723.

Kirk, C., Lewis-Moss, R., Nilsen, C. \& Colvin, D. (2011). The Role of Parent Expectations on Adolescent Educational Aspirations. Educational Studies, 37(1), 89-99.

Lam, S., Chow-Yeung, K., Wong, B., Lau, K. \& Tse, S. (2013). Involving Parents in Paired Reading with Preschoolers: Results from a Randomized Controlled Trial. Contemporary Educational Psychology, (38), 126-135.

López, O., Hederich, C. \& Camargo, Á. (2011, enero-abril). Estilo cognitivo y logro académico. Educación y Educadores, 14(1), 67-82.

Ma, X. (2005). Growth in Mathematics Achievement: Analysis with Classification and Regression Trees. The Journal of Educational Research, 99(2), 78-86.

Mancebón-Torrubia, M. J., Calero, J., Choi, Á. \& Ximénez-de-Embún, D. (2010). The Efficiency of Public and Publicly Subsidized High Schools in Spain: Evidence from PISA-2006. Journal of the Operational Research Society, 63(11), 1516-1533.

Maslow, A. (1943). A Theory of Human Motivation. Psychological Review, (50), 370-396.

Meckes, L. (2007). Evaluación y estándares: logros y desafíos para incrementar el impacto en calidad educativa. Revista Pensamiento Educativo, 40(1), 351-371. 
Mendez, J. (2010). How Can Parents Get Involved in Preschool? Barriers and Engagement in Education by Ethnic Minority Parents of Children Attending Head Start. Cultural Diversity and Ethnic Minority Psychology, (16), 26-36.

Milford, T., Shelley, P. \& Anderson, J. O. (2010). An Opportunity to Better Understand Schooling: The Growing Presence of Pisa in the Americas. International Journal of Science and Mathematics Education, 8(3), 453-473.

mineduc. (2012). Informe Técnico Simce 2012. Agencia de Calidad de la Educación. Santiago de Chile: Ministerio de Educación de Chile. Recuperado de https://s3-us-west-2.amazonaws. com/documentos-web/Informe_Tecnico_Simce_2012.pdf

MINEDUC. (2011). Informe de Resultados Nacionales Simce. Unidad de Currículum y Evaluación. Santiago de Chile: Ministerio de Educación de Chile.

MINEDUC. (2010). Resumen resultados PISA, Chile 2009. Unidad de Curriculum y Evaluación. Santiago de Chile: Ministerio de Educación de Chile.

MINEDUC. (2009). ¿Qué nos dice PISA sobre la educación de los jóvenes en Chile? Unidad de Currículum y Evaluación. Santiago de Chile: Ministerio de Educación de Chile.

mineduc. (2002). Prueba Simce $2^{\circ}$ medio 2001: Factores que inciden en el rendimiento de los alumnos. Santiago de Chile: Departamento de Estudios y Estadísticas.

Mizala, A. \& Romaguera, P. (2000). Determinación de factores explicativos de los resultados escolares en educación media en Chile. Serie Economía, (85).

Mizala, A. \& Torche, F. (2012). Bringing the Schools Back in: The Stratification of Educational Achievement in the Chilean Voucher System. International Journal of Educational Development, 32(1), 132-144.

Mogonea, F. \& Mogonea, F. (2014). The Role of the Family in Building Adolescents' Self-esteem. Procedia-Social and Behavioral Sciences, (127), 189-193.

Moreira, P., Dias, P., Machado, F. \& Machado, J. (2013). Predictors of Academic Performance and School Engagement-Integrating persistence, Motivation and study Skills Perspectives Using Person-centered and Variable-centered Approaches. Learning and Individual Differences, (24), 117-125.

Murillo, F. (2007). Resultados de aprendizaje en América Latina a partir de las evaluaciones nacionales. UNESCO/OREALC. 
Murillo, J. \& Román, M. (2011). ¿¿La escuela o la cuna? Evidencias sobre su aportación al rendimiento de los estudiantes de América Latina. Estudio multinivel sobre la estimación de los efectos escolares. Profesorado, 15(3), 27-50.

OECD. (2012). Economic Surveys: Chile 2012. París: Organization for Economic Cooperation and Development Publishing.

OECD. (2009). Top of the Class. High Performers in Science in PISA 2006. París: Organization for Economic Cooperation and Development Publishing.

OECD. (2007). El programa PISA de la OECD; Qué es y para qué sirve? París: Organization for Economic Cooperation and Development Publishing.

Paredes, R. \& Pinto, J. (2009). Is this the End of Public Education In Chile? Estudios de Economía, 36(1), 47-66.

Phillipson, S. \& Phillipson, N. (2007). Academic Expectations, Belief of Ability, and Involvement by Parents as Predictors of Child Achievement: A Cross Cultural Comparison. Educational Psychology: An International Journal of Experimental Educational Psychology, 27(3), 329-348.

Räty, H. (2006). What Comes after Compulsory Education? A Follow-up Study on Parental Expectations of their Child's Future Education. Educational Studies, 32(1), 1-16.

Reyes, J., Escobar, C., Duarte, J. \& Ramírez, P. (2007). An Application of the Model Logistic Regression in the Prediction of the Student Performance. Estudios Pedagógicos, 33(2), 101-120.

Salvo, S., Moraga, A. Miranda, H., Ramírez, M. \& Vera, D. (2012). Avanzando en la identificación de factores que explican un mejor rendimiento escolar en estudiantes y establecimientos chilenos, en las áreas de Matemática, Ciencias y Lenguaje: propuesta para el diseño de políticas públicas. FONIDE FP111007.

Schiattino, I. \& Silva, C. (2008). Árboles de clasificación y regresión: Modelos CART. Ciencia \& Trabajo, 10(30), 61-166.

Taut, S., Cortes, C., Sebastian, C. \& Preiss, D. (2009). Evaluating School and Parent Reports of the National Student Achievement Testing System (Simce) in Chile: Access, Comprehension, and Use. Evaluation and Program Planning, 32(2), 129-137.

Tomopoulos, S., Dreyer, B., Tamis-LeMonda, C., Flynn, V., Rovira, I., Tineo, W. \& Mendelsohn, A. (2006). Books, Toys, Parent-Child Interaction, and Development in Young Latino Children, Ambulatory Pediatrics, 6(2), 72-78. 
Valenzuela, J. P., Bellei, C. \& De los Ríos, D. (2008). Evolución de la Segregación Socioeconómica de los Estudiantes Chilenos y su Relación con el Financiamiento Compartido. Proyecto FONIDE 211-2006.

Villalobos, C. \& Valenzuela, J. P. (2012). Polarización y cohesión social del sistema escolar chileno. Revista de Análisis Económico, 27(2), 145-172.

Villiger, C., Niggli, A., Wandeler, C. \& Kutzelmann, S. (2012). Does Family Make a Difference? Mid-term Effects of a School/Home-based Intervention Program to enhance Reading Motivation. Learning and Instruction, (22), 79-91.

Vryonides, M. \& Gouvias, D. (2012). Parents' Aspirations for their Children's Educational and Occupational Prospects in Greece: The Role of Social Class. International Journal of Educational Research, (53), 319-329.

Wormington, S., Corpus, J. \& Anderson, K. (2012). A Person-centered Investigation of Academic Motivation and its Correlates in High School. Learning and Individual Differences, 22(4), 429-438.

Yoo, B. \& Donthu, N. (2001). Developing a Scale to Measure the Perceived Quality of an Internet Shopping Site (sITEQUAL). Quarterly Journal of Electronic Commerce, 2(1), 31-47.

Zorrilla, M. (2008). La escuela secundaria mexicana. Un estudio multinivel de los efectos escolares y sus propiedades cientificas. Tesis doctoral. Universidad Anáhuac. México.

Recibido el 10 de octubre de 2014.

Aceptado el 1 de junio de 2016. 